\title{
MicroRNA expression profile of HCT-8 cells in the early phase of Cryptosporidium parvum infection
}

\author{
Chenrong Wang ${ }^{1,2}$, Limin Liu ${ }^{1,2}$, Huili Zhu ${ }^{3}$, Lu Zhang ${ }^{1,2}$, Rongjun Wang ${ }^{1,2}$, Zhenjie Zhang ${ }^{1,2}$, Jianying Huang ${ }^{1,2}$, \\ Sumei Zhang ${ }^{1,2}$, Fuchun Jian ${ }^{1,2}$, Changshen Ning ${ }^{1,2}$ and Longxian Zhang ${ }^{1,2^{*}}$ (D)
}

\begin{abstract}
Background: Cryptosporidium parvum is an important zoonotic parasitic disease worldwide, but the molecular mechanisms of the host-parasite interaction are not fully understood. Noncoding microRNAs (miRNAs) are considered key regulators of parasitic diseases. Therefore, we used microarray, $\mathrm{QPCR}$, and bioinformatic analyses to investigate the intestinal epithelial miRNA expression profile after Cryptosporidium parvum infection.

Results: Twenty miRNAs were differentially expressed after infection (four upregulated and 16 downregulated). Further analysis of the differentially expressed miRNAs revealed that many important cellular responses were triggered by Cryptosporidium parvum infection, including cell apoptosis and the inflammatory and immune responses.

Conclusions: This study demonstrates for the first time that the miRNA expression profile of human intestinal epithelium cells is altered by C. parvum infection. This dysregulation of miRNA expression may contribute to the regulation of host biological processes in response to C. parvum infection, including cell apoptosis and the immune responses. These results provide new insight into the regulatory mechanisms of host miRNAs during cryptosporidiosis, which may offer potential targets for future C. parvum control strategies.
\end{abstract}

Keywords: Cryptosporidium parvum, Intestinal epithelium, Infection, MicroRNA

\section{Background}

Cryptosporidium is a genus of protozoan parasites that infect the gastrointestinal epithelium and other mucosal surfaces of their hosts, which include humans and domestic and wild animals worldwide [1-3]. Cryptosporidium is a major cause of moderate to severe diarrhea in children younger than 2 years, particularly infants, and is second only to rotavirus in this regard [4]. Morbidity and mortality are associated with cryptosporidial infections in patients with acquired immunodeficiency syndrome (AIDS) and young children $[1,5]$. Cryptosporidium parvum is one of the species most commonly involved in human cryptosporidial infections [1]. Despite intensive efforts over the past 30 years, the precise molecular

\footnotetext{
* Correspondence: zhanglx8999@gmail.com

${ }^{1}$ College of Animal Science and Veterinary Medicine, Henan Agricultural University, Zhengzhou 450002, People's Republic of China

${ }^{2}$ International Joint Research Laboratory for Zoonotic Diseases of Henan, Zhengzhou 450002, People's Republic of China

Full list of author information is available at the end of the article
}

pathogenic mechanisms of cryptosporidial infection are not fully understood.

MicroRNAs (miRNAs) are endogenous noncoding small RNAs (22 nucleotides) that regulate gene expression at the posttranscriptional level and play important roles in the regulation of diverse pathological processes, including cell proliferation, differentiation, metabolism, the immune response, and apoptosis [6-8]. The miRNAs in mammalian cells have been shown to play crucial roles in the cellular responses to infection by diverse pathogens, including viruses, parasites, and bacteria [9-11]. Increasing evidence suggests that host miRNAs help the host to clear parasites by regulating the toll-like receptor 4 (TLR4) signaling pathways and the release of antimicrobial peptides $[12,13]$. For example, C. parvum infection reduces the expression of the let-7 family miRNAs in biliary epithelial cells, which increases SNAP23 expression, coordinating the release of exosomes carrying antimicrobial-peptide in response to C. parvum infection [13]. The upregulation of

(c) The Author(s). 2019 Open Access This article is distributed under the terms of the Creative Commons Attribution 4.0 International License (http://creativecommons.org/licenses/by/4.0/), which permits unrestricted use, distribution, and reproduction in any medium, provided you give appropriate credit to the original author(s) and the source, provide a link to the Creative Commons license, and indicate if changes were made. The Creative Commons Public Domain Dedication waiver (http://creativecommons.org/publicdomain/zero/1.0/) applies to the data made available in this article, unless otherwise stated. 
miR-27b expression enhances the anti-C. parvum responses of biliary epithelial cells [14]. Some evidence suggests that host miRNAs are also used by C. parvum to enhance its own survival. For example, suppressor of cytokine signaling (SOCS) proteins are negative regulators of cytokine signaling [15]. Cryptosporidium parvum infection downregulates the expression of miR-98 and let-7 to induce SOCS protein expression in biliary epithelial cells [16]. The upregulation of miR-21 after C. parvum infection inhibits the activation of TLR4/NF- $\mathrm{KB}$ signaling in biliary epithelial cells by targeting PDCD4, a proinflammatory protein that promotes the activation of NF- $\mathrm{KB}$ [17]. Therefore, studying the changes in the host miRNA expression profile after $C$. parvum infection will extend our understanding of the interaction between $C$. parvum and its host.

There has been no report of the changes in the miRNA expression profile in the intestinal epithelium after C. parvum infection. Therefore, the objective of this study was to analyze the intestinal epithelial miRNA expression profile after C. parvum infection using microarray and bioinformatics analyses in an in vitro model using human ileocecal HCT-8 adenocarcinoma cells.

\section{Methods}

\section{Cell and parasite}

Human ileocecal adenocarcinoma (HCT-8) cells (American Type Culture Collection, Manassas, VA) were cultured and maintained in Dulbecco's modified Eagle's medium (DMEM) supplemented with $10 \%$ fetal bovine serum, $4 \mathrm{mmol} / \mathrm{L} \mathrm{L}$-glutamine, $100 \mathrm{U} / \mathrm{mL}$ penicillin, and $100 \mathrm{U} / \mathrm{mL}$ streptomycin at $37^{\circ} \mathrm{C}$ in a humidified $5 \% \mathrm{CO}_{2}$ incubator.

Neonatal calves were purchased from Ruiya Animal Husbandry Co., Ltd. (Zhengzhou, China). Oocysts of $C$. parvum (IId subtype) were maintained in infected neonatal calves, purified from their feces, and stored at $4{ }^{\circ} \mathrm{C}$ in $2.5 \%$ potassium dichromate until use. Before use, the oocysts were washed with phosphate-buffered saline (PBS), treated with $10 \%$ hypochlorite for $10 \mathrm{~min}$ at $4{ }^{\circ} \mathrm{C}$, and washed again [18]. The infected calf was killed by anesthesia (113 mg sodium pentobarbital $/ \mathrm{kg}$, Intravenous injection) and carcass was treated harmlessly by a specialized agency of our college.

The infection assays have been reported previously [18]. Briefly, cell monolayers in six-well cell culture dishes were inoculated with $100 \mu \mathrm{L}$ of purified oocysts $\left(1 \times 10^{7} /\right.$ well $)$ in DMEM, that the oocyst:host cell ratio is 5:1. Heat-inactivated $\left(100^{\circ} \mathrm{C}, 5 \mathrm{~min}\right)$ oocysts were used as the controls. After $2.5 \mathrm{~h}$, oocysts and sporozoites remaining in the supernatant were removed and replaced with DMEM containing $2 \%$ fetal bovine serum, $4 \mathrm{mmol} / \mathrm{L} \mathrm{L}$-glutamine, $100 \mathrm{U} / \mathrm{mL}$ penicillin, $100 \mathrm{~g} / \mathrm{mL}$ streptomycin, $0.3 \mathrm{~g} / \mathrm{L}$ bicarbonate, and $0.02 \mathrm{~g} / \mathrm{L}$ taurocholate. The plates were incubated at $37^{\circ} \mathrm{C}$ in a humidified $5 \% \mathrm{CO}_{2}$ incubator.

\section{Total RNA isolation and quality control}

At 4 and $12 \mathrm{~h}$ postinfection, the cells were washed three times with PBS, and $1 \mathrm{~mL}$ of TRIzol Reagent (Invitrogen, Carlsbad, CA, USA) was added to each well. The total RNA from three uninfected separate monolayers and three infected separate monolayers were isolated according to the manufacturer's protocol.

The quality of the RNA was measured with a NanoDrop 2000 spectrophotometer (Thermo Fisher Scientific, Inc., Waltham, MA, USA). RNA purity was assessed from the absorbance (A) of the solutions at various wavelengths: $2.0>\mathrm{A}_{260} / \mathrm{A}_{280}>1.8$ and $\mathrm{A}_{260} / \mathrm{A}_{230}>2.0$ were considered to indicate high purity. RNA integrity was determined from the 28S:18S rRNA ratio determined with an electrophoretic analysis.

\section{Microarray analysis}

miRNA microarrays based on miRBase v20.0 (http:// www.mirbase.org/) were used to study the expression profiles of 2555 mature miRNAs. Microarray chips are synthesized by LC Sciences and have patent rights. The chips are made by a flexible, in situ synthesis method using conventional chemistry. The technology encompasses a novel high-throughput biopolymer synthesis chemistry carried out using a new class of microfluidic reaction devices and an advanced digital light synthesizer apparatus and is developed for carrying out picoliter scale chemical and/or biochemical reactions. Briefly, Hybridization was performed using the $\mu$ Paraflo $^{\circ}$ microfluidic array technology (LC Sciences, Hangzhou, China), according to the manual. The assay started from 4 to $8 \mu \mathrm{g}$ total RNA sample were 3 -extended with a poly(A) tail using poly(A) polymerase. An oligonucleotide tag that binding to Cy3 dyes in later chip hybridization was then ligated to the poly(A) tail. Hybridization was performed overnight on a $\mu$ Paraflo microfluidic chip using a micro-circulation pump (Atactic Technologies). On the microfluidic chip, each detection probe consisted of a chemically modified nucleotide coding segment complementary to target microRNA (from miRBase, http://www.mirbase.org/). The detection probes were made by in situ synthesis using PGR (photogenerated reagent) chemistry. The hybridization melting temperatures were balanced by chemical modifications of the detection probes. Hybri dization used $100 \mu \mathrm{L}$ 6xSSPE buffer $(0.90 \mathrm{M} \mathrm{NaCl}, 60$ $\mathrm{mM}$ Na2HPO4, $6 \mathrm{mM}$ EDTA, pH 6.8) containing 25\% formamide at $34^{\circ} \mathrm{C}$. After RNA hybridization, oligonucleotide tag-conjugating $\mathrm{Cy} 3$ dye were circulated through the microfluidic chip for dye staining. Fluorescence images were collected using a laser scanner 
(GenePix 4000B, Molecular Device) and digitized using Array-Pro image analysis software (Media $\mathrm{Cy}$ bernetics). Data were analyzed by first subtracting the background and then normalizing the signals using a LOWESS filter (Locally-weighted Regression).

Each miRNA has a fold change in the signals detected in infected cells relative to the signals detected in uninfected cells and the $P$ value that calculated with Student's $t$ test. Differentially expressed miRNAs was selected based on a fold change of $>2$ or $<-2$ and a $P$ value of $\leq 0.05$.

\section{Validation of microarray data with quantitative PCR (qPCR) analysis}

miRNAs were prepared with an All-in-One ${ }^{\mathrm{Tm}}$ miRNA qRT-PCR Detection Kit (GeneCopoeia, Rockville, MD, USA), according to the manufacturer's instructions. Briefly, the extracted RNA was reverse-transcribed in the presence of a poly(A) polymerase and an oligo-dT adaptor. Quantitative PCR was then performed with SYBR Green detection using a forward primer specific for the mature miRNA sequence and a universal adaptor reverse primer. The forward primers used for the qPCRs are shown in Table 1. Each RNA was run in triplicate wells with three biological replicates, including three uninfected separate monolayers and three infected separate monolayers. The cycling protocol was: $95^{\circ} \mathrm{C}$ for $10 \mathrm{~min}$, followed by 40 cycles of $95^{\circ} \mathrm{C}$ for $10 \mathrm{~s}$ and $60^{\circ} \mathrm{C}$ for $20 \mathrm{~s}$, with a final extension at $72{ }^{\circ} \mathrm{C}$ for $20 \mathrm{~s}$. When all cycles were finished, a melting-curve analysis was performed. The baseline and cycle threshold $(\mathrm{Ct})$ values were automatically determined for all plates with the Roche LightCycler 96 Real-Time PCR System. The miRNA expression levels in each sample were normalized to the expression of the housekeeping gene U6 [19-21]. The real-time PCR results were analyzed and expressed as the relative $\mathrm{Ct}$ value using the $2^{-\Delta \Delta \mathrm{Ct}}$ method [22]. The mean and standard deviation $(\mathrm{M} \pm \mathrm{SD})$ was calculated from three independent biological trials. Differences between infected and control group were measured using the t-test by SPSS 17.0 with $P<0.05$ indicating significance.

Table 1 Specific primers used for $\mathrm{qPCR}$

\begin{tabular}{ll}
\hline miRNAs & Forward primer \\
\hline hsa-miR-122-5p & TGGAGTGTGACGATGGTGTTTG \\
hsa-miR-3591-3p & AGCATCACCATTGTCACACTCCAC \\
hsa-miR-5580-3p & CACAGTTGAAGAGAGCCAGCAC \\
hsa-miR-181d-3p & TCATAGGGGGCTCAATGTCAC \\
hsa-miR-454-5p & CGCCTATCGCTATTGTCTCTGC \\
hsa-let-7b-3p & GCATACAACCTACTGCCTCCC \\
U6 & TGGCAAGGATGACACGCAAAT \\
\hline
\end{tabular}

Predicted target genes of differentially expressed miRNAs, gene ontology (GO) and Kyoto encyclopedia of genes and genomes

\section{(KEGG) pathway analyses}

Despite an abundance of online software for predicting human microRNA targets, many human miRNAs are only included in TargetScanHuman7.1 and miRDB. Therefore, the target genes of the miRNAs were predicted with the two online programs TargetScanHuman7.1 (http://www.targetscan.or g/vert_71/) [23] and miRDB (http://www.mirdb.org/cgi-bin/ search.cgi) [24]. The target genes of the differentially expressed miRNAs were analyzed in terms of their GO categories and KEGG pathways using the Database for Annotation, Visualization and Integrated Discovery (DAVID) (https://david.ncifcrf.gov/), a gene annotation tool [25].

\section{miRNA-target gene network analysis}

The miRNA- predicted target genes regulatory network was constructed with the miRNA-target gene pairs using the Cytoscape software(www.cytoscape.org) [26-28], based on the interactions of the miRNAs and genes [29], Briefly, a text file containing miRNAs and predicted target genes was imported in Cytoscape software and optimized the visual features of nodes and edges, such as shape and color. With an miRNA-target gene network, we can identify the crucial miRNA which have the highest connectivity to other miRNAs. The higher the connectivity of a miRNA was, the more important it was in the network. Also, we can find the key genes which were the common target genes of the differentially expressed miRNAs.

\section{Results}

Differentially expressed miRNAs

A total of 2555 mature human miRNA sequences were detected in C. parvum-infected and uninfected HCT-8 cells using miRNA microarrays (Sanger miRBase v20.0). A dendrogram produced with a hierarchical clustering analysis of the miRNAs differentially expressed in $C$. parvum-infected and -uninfected cells is shown in Fig. 1. In total, 20 miRNAs (four upregulated and 16 downregulated) were significantly differentially expressed in the infected HCT-8 cells compared to uninfected cells. These differentially expressed miRNAs (with fold changes $\geq 2$ and $P \leq 0.05)$ are shown in Table 2 with $P$ values. From this table, it is clear that downregulated miRNAs were more frequent than upregulated miRNAs $(P<0.05)$. Among these miRNAs, hsa-miR-942-5p was most strongly upregulated and hsa-miR-4689 was most strongly downregulated.

\section{Validation of miRNA microarray data with qPCR}

Six selected miRNAs and the housekeeping U6 mRNA were assayed with $\mathrm{qPCR}$ to confirm the expression 

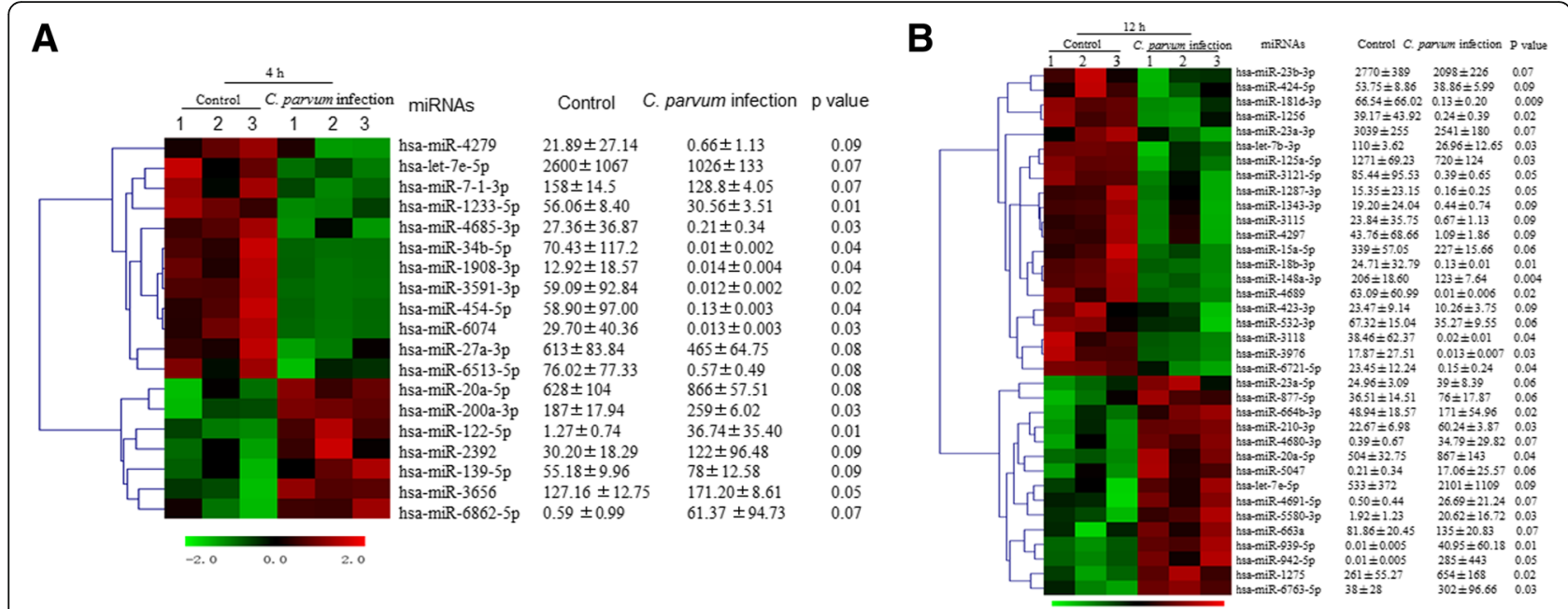

Fig. 1 Expression profiling of miRNAs in HCT-8 cells after C. parvum infection. Expression profiles of miRNAs in HCT-8 cells at $4 \mathrm{~h}$ after $\mathrm{C}$. parvum infection. The horizontal axis indicates samples of non-infected cells $(n=3$; Control 1,2 , and 3$)$ and cells after exposure to live $C$. parvum for $12 \mathrm{~h}$ $(\mathrm{n}=3$, C. parvum 1, 2, and 3) (a). Expression profiles of miRNAs in HCT-8 cells at $12 \mathrm{~h}$ after $\mathrm{C}$. parvum infection. The horizontal axis indicates samples of non-infected cells ( $n=3$; Control 1, 2, and 3) and cells after exposure to live C. parvum for $12 h(n=3$, C. parvum 1, 2, and 3) (b)

Table 2 Differentially expressed miRNAs in the infected HCT-8 cells compared to uninfected cells

\begin{tabular}{llll}
\hline Infection time & hsa-microRNAs & Log2 fold change & $P$-value \\
\hline $4 \mathrm{~h}$ & hsa-miR-122-5p & +4.85 & 0.01 \\
& hsa-miR-3591-3p & -12.29 & 0.02 \\
& hsa-miR-6074 & -11.12 & 0.03 \\
& hsa-miR-454-5p & -12.16 & 0.04 \\
& hsa-miR-34b-5p & -12.19 & 0.04 \\
& hsa-miR-4685-3p & -7.02 & 0.03 \\
12h & hsa-miR-1908-3p & -9.88 & 0.04 \\
& hsa-miR-942-5p & +15.26 & 0.05 \\
& hsa-miR-5580-3p & +3.42 & 0.03 \\
& hsa-miR-6763-5p & +2.99 & 0.03 \\
& hsa-miR-181d-3p & -8.99 & 0.009 \\
& hsa-miR-18b-3p & -10.94 & 0.01 \\
& hsa-miR-4689 & -12.30 & 0.02 \\
& hsa-miR-1256 & -7.35 & 0.02 \\
hsa-miR-3976 & -10.42 & 0.03 \\
hsa-let-7b-3p & -2.09 & 0.03 \\
hsa-miR-6721-5p & -7.29 & 0.04 \\
hsa-miR-3118 & -11.23 & 0.04 \\
hsa-miR-3121-5p & -7.77 & 0.05 \\
hsa-miR-1287-3p & -6.62 & 0.05 \\
\hline
\end{tabular}

* "+"represents up-regulation, "-"represents down-regulation profiles identified with the microarray analysis. The expression patterns determined with qPCR agreed well with those determined from the microarray data (Fig. 2).

\section{Predicting the target genes of differentially expressed miRNAs}

We predicted the target genes for all the miRNAs differentially expressed in HCT-8 cells after C. parvum infection. Target genes were predicted for only 18 of the 20 differentially expressed miRNAs using two different online software programs, TargetScan and miRDB.

\section{GO analysis of differentially expressed miRNAs}

To further understand the functions of the differentially expressed miRNAs, a GO analysis of the target genes was performed. DAVID gene annotation was applied to explain the biological effects of the differentially expressed miRNAs based on their target genes. The GO annotations of differentially expressed miRNAs (with $P$ $<0.05)$ are shown in Fig. 3. The GO-specific functions mainly involved biological processes (e.g., transcription initiation from the RNA polymerase II promoter, apoptotic process, and immune response), cell components (e.g., membrane, actin cytoskeleton, focal adhesion, plasma membrane, cell surface, and apical part of the cell), and molecular functions (e.g., protein binding, ATP binding, calcium ion binding). Among the GO annotations (with $P<0.05$ ), hsa-miR-34b-5p, hsa-miR-942-5p, hsa-miR-3591-3p, hsa-miR-18b-3p and hsa-miR-3976 were involved in the regulation of apoptotic or autophagy processes, hsa-miR-34b-5p and hsa-miR-3591-3p were involved in the regulation of the immune response (Additional file 1: Figure S1). Thus, the GO analysis of 

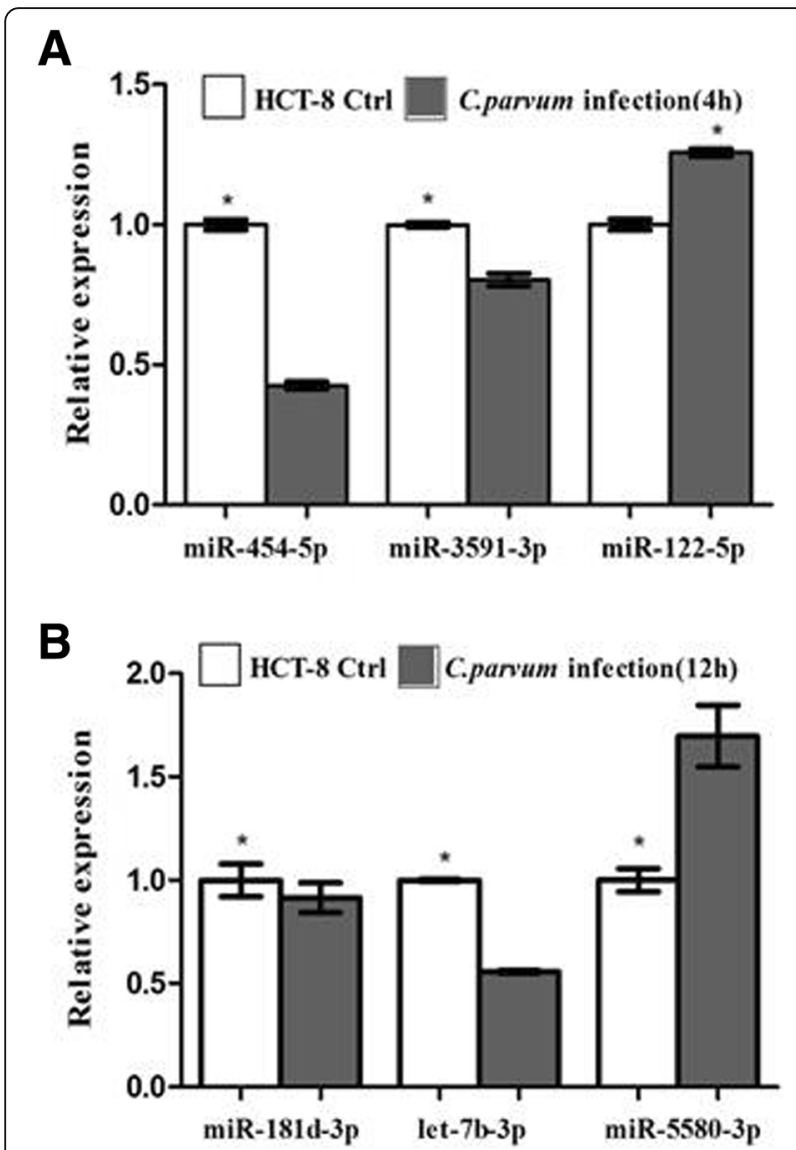

Fig. 2 qPCR confirmation of the miRNA microarray data. $\mathrm{QPCR}$ confirmation of the miRNAs differentially expressed at $4 \mathrm{~h}$ after $C$. parvum infection (a). qPCR confirmation for the miRNAs differentially expressed at $12 \mathrm{~h}$ after C. parvum infection (b). Data are the means and standard errors of the means of triplicate experiments

the differentially expressed miRNA target genes revealed that some have potentially important biological functions in regulating the host against C. parvum infection.

\section{KEGG pathway analysis of differentially expressed miRNAs}

The main KEGG pathway annotations of the target genes (with $\mathrm{P}<0.05$ ) are shown in Table 3. Among these KEGG pathways, several were associated with the immune response (e.g., cytokine receptor interaction, chemokine signaling pathway, and inflammatory mediator regulation of TRP channels), apoptosis (e.g., MAPK signaling pathway and ERBB signaling pathway), cytoskeleton (e.g., actin cytoskeleton and WNT signaling pathway), and cell adhesion (e.g., cell adhesion molecules).

\section{Regulatory network of miRNAs and target genes}

To investigate the interactions between the miRNAs and their target genes, we performed an miRNA-target gene network analysis (Fig. 4). From the network of the
miRNA and their target genes at $4 \mathrm{~h}$ after C. parvum infection (Fig. 4a), we found among seven differentially expressed miRNAs at $4 \mathrm{~h}$ hsa-miR-34b-5p and hsa-miR3591-3p have a connection with six differentially expressed miRNAs. Among eleven differentially expressed miRNAs at $12 \mathrm{~h}$, hsa-miR-942-5p, hsa-let-7b-3p and hsa-miR-6721-5p have a connection with ten differentially expressed miRNAs(Fig. 4b). According to these datasets, the key miRNAs among the differentially expressed miRNAs were hsa-miR-34b-5p, hsa-miR-3591-3p, hsa-miR942-5p, hsa-let-7b-3p, and hsa-miR-6721-5p Their common target genes of the key miRNAs at $12 \mathrm{~h}$ were shown in Fig. 4c.

\section{Discussion}

Increasing evidence suggests that miRNAs regulate gene expression at the posttranscriptional level and thus affect a variety of biological processes, including the pathogenesis and progression of various diseases [30]. miRNAs also play important roles in the regulation of complex parasite-host interactions [10,12, 14, 15]. Cryptosporidium parvum-induced miRNA expression in human biliary epithelial cells has already been investigated and analyzed [10]. However, there has been no report of the miRNA expression profile of human intestinal epithelial cells infected with C. parvum. Therefore, we analyzed the changes in the miRNA the profile of HCT-8 cells at $4 \mathrm{~h}$ and $12 \mathrm{~h}$ after C. parvum infection using microarray technology. Our results show that most miRNAs were not significantly differentially expressed in the infected HCT-8 cells compared to uninfected cells. However, some differentially expressed miRNAs were detected (four upregulated and 16 downregulated), implying that C. parvum infection alters the miRNA profile of intestinal epithelial cells. However, the differentially expressed miRNAs in the infected HCT-8 cells differed from those in biliary epithelial cells [10]. The changes in these specific miRNAs induced by C. parvum might be involved in the regulation of human intestinal epithelial cells in response to $C$. parvum infection. This is the first report to describe the alterations in miRNA expression induced by $C$. parvum infection in human intestinal epithelial cells, and extends our understanding of the interplay between $C$. parvum and its host at the miRNA level. The identification of the functions of these miRNA sheds new light on the molecular mechanisms triggered by C. parvum infection. Therefore, in this study, GO and KEGG analyses were used to identify the functions of the predicted target genes involved in C. parvum infection. Cell apoptosis is one of the ancient antiparasite strategies used by a host after its invasion by a parasite [31-34]. Research has shown that the early phase of $C$. parvum infection results in the apoptosis of HCT-8 cells [35]. However, miRNA-mediated cell apoptosis after $C$. 


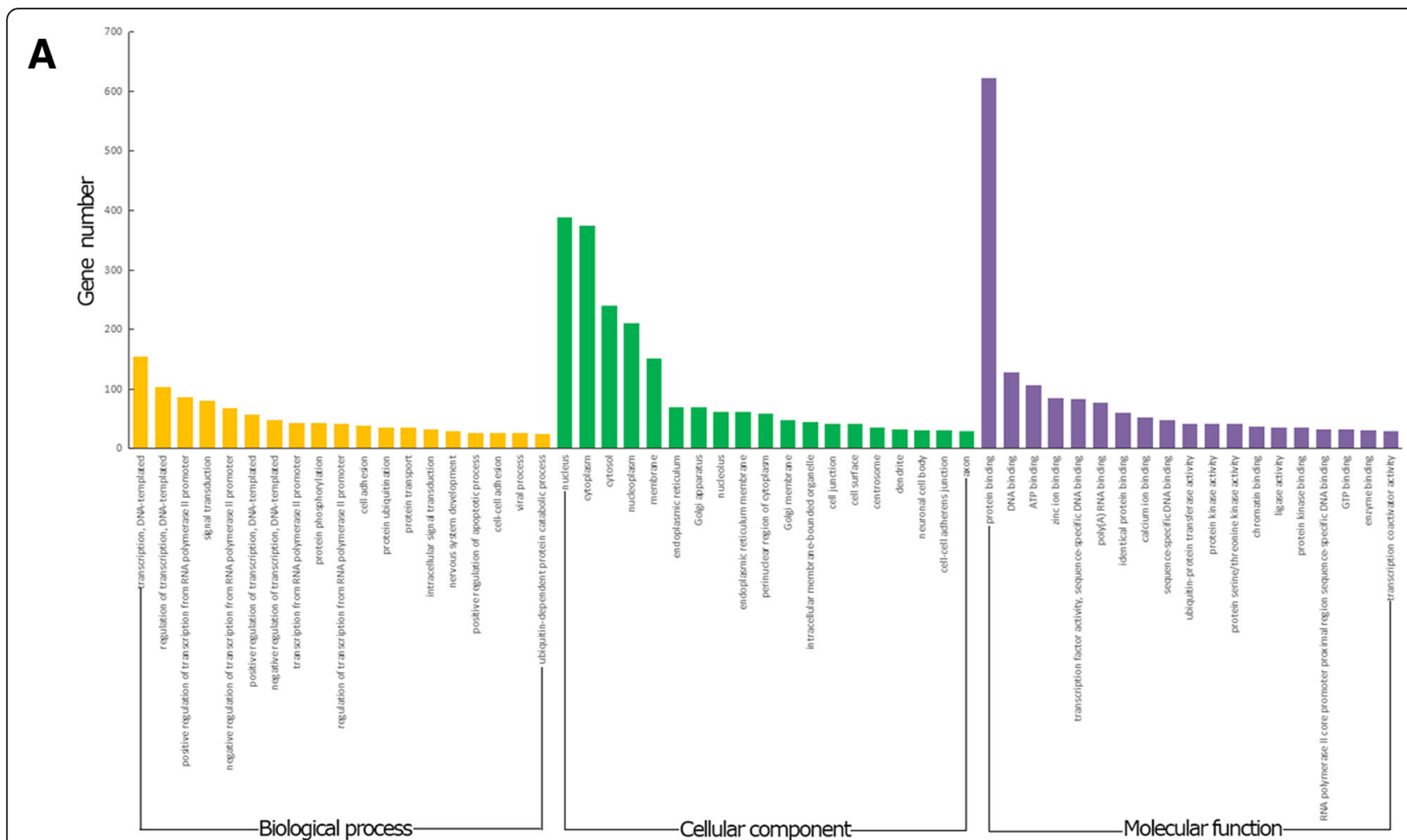

B

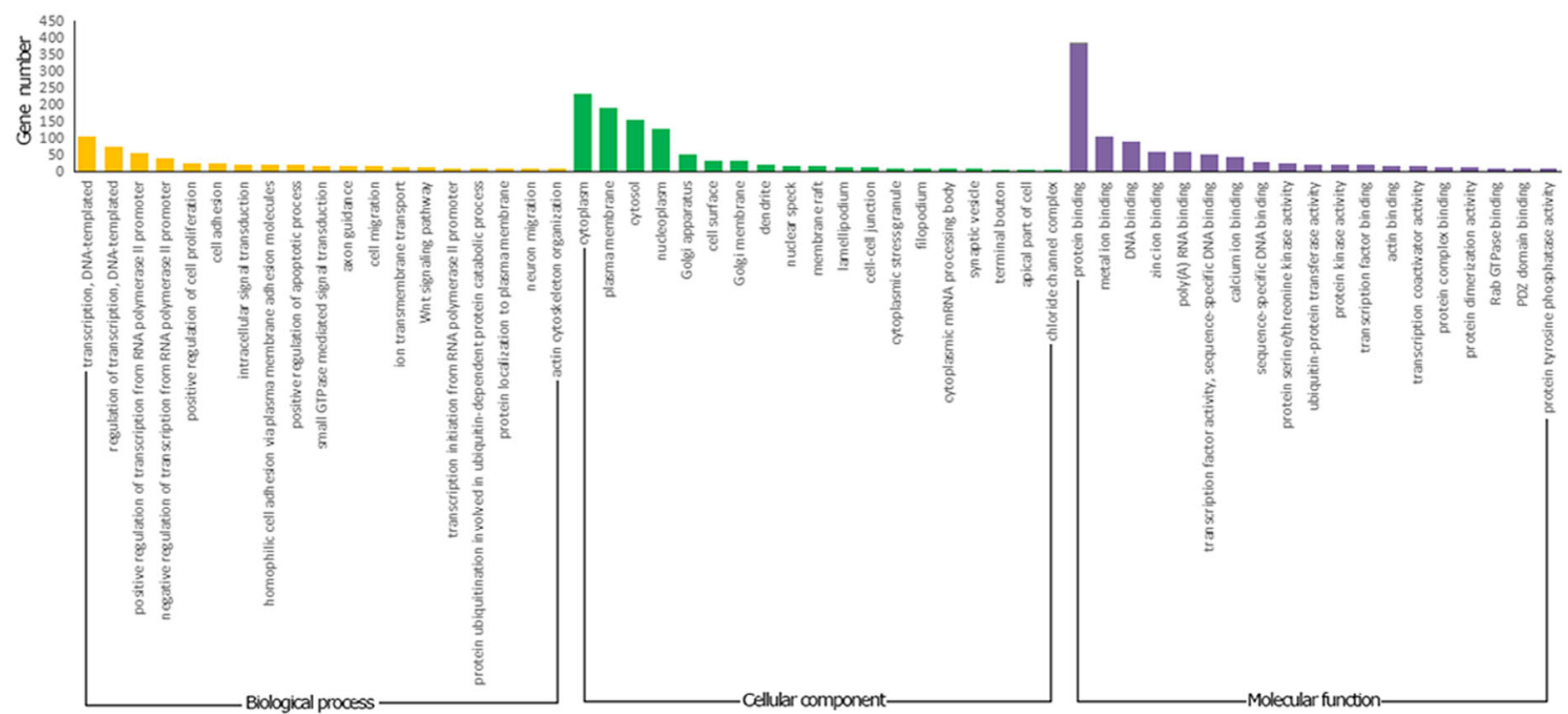

Fig. $3 \mathrm{GO}$ analysis of targets of differentially expressed miRNAs at $4 \mathrm{~h}$ and $12 \mathrm{~h}$ respectively. According to gene count, top $19 \mathrm{GO}$ terms $(P<0.05)$ are shown. GO analysis of targets of differentially expressed miRNAs at $4 \mathrm{~h}(\mathbf{a})$. GO analysis of targets of differentially expressed miRNAs at $4 \mathrm{~h}$ (b)

parvum infection has not been reported. Our results suggest that the functions of hsa-miR-34b-5p, hsa-miR942-5p, hsa-miR-18b-3p, hsa-miR-3976 and hsa-miR3591-3p, most of which (not hsa-miR-942-5p) were downregulated after C. parvum infection, regulate apoptotic processes. Several reports have shown that hsamiR-34b, hsa-miR-18a, and hsa-miR-942 regulate cell apoptosis in response to microbial infection. For example, upregulated hsa-miR-18a in macrophages after
Toxoplasma gondii infection leads to the survival of the infected macrophages by targeting BIM [36]. Upregulated hsa-miR-30c-1 in human macrophages is involved in an anti-apoptosis response to T. gondii infection [37]. The downregulation of hsa-miR-34a induces cell apoptosis in Influenza A virus-infected A549 cells [38], and the downregulation of hsa-miR-942 enhances the apoptosis of HLCZ01 cells in response to Hepatitis $C$ virus infection [39]. 
Table 3 KEGG pathways of differentially expressed miRNAs

\begin{tabular}{|c|c|c|c|}
\hline Hsa-miRNA & Pathway & Gene & $P$ value \\
\hline \multirow[t]{4}{*}{ miR-34b-5p } & Ubiquitin mediated proteolysis & CBLB, CBL, NHLRC1, BTRC, NEDD4, PIAS1 & 0.0073 \\
\hline & AMPK signaling pathway & ELAVL1, RAB10, RAB14, CREB1, HNF4, APRKA2 & 0.04 \\
\hline & Notch signaling pathway & APH1A, DLL1, JAG1, NOTCH1, NOTCH2 & 0.017 \\
\hline & Hippo signaling pathway & MOB1B, WNT2B, BTRC, BMPR2, DLG1, GSK3B & 0.013 \\
\hline \multirow[t]{6}{*}{ miR-942-5p } & Ras signaling pathway & RAF1, TEK, CHUK, FGF9MAPK10, MRAS... & 0.006 \\
\hline & ErbB signaling pathway & RAF1, CDKN1B, MAPK10, NRAS, PAK2, PIK3CD & 0.0082 \\
\hline & MAPK signaling pathway & RAF1, TAOK1, TAB2, CACNA1E, CHUK, .. & 0.014 \\
\hline & Wnt signaling pathway & CREBBP, FBXW11, WNT9B, CCND1... & 0.022 \\
\hline & p53 signaling pathway & CHEK1, CCND1, RFWD2, SERPINE1, ZMAT3 & 0.046 \\
\hline & PI3K-Akt signaling pathway & MCL1, RAF1, TEK, COL4A5, CHUK, CCND1... & 0.047 \\
\hline \multirow[t]{3}{*}{ miR-3591-3 } & Ubiquitin mediated proteolysis & CBLHERC3, CDC27, CUL5, MAP3K1, RFWD2... & 0.0049 \\
\hline & ErbB signaling pathway & SOS2, CAMK2A, MAP2K4, NRG3, STAT5B & 0.016 \\
\hline & MAPK signaling pathway & ELK4, SOS2, TAB2, DAXX, FGF14, MAP2K3... & 0.017 \\
\hline \multirow[t]{4}{*}{ miR-6721-5p } & Cell adhesion molecules (CAMs) & CD22, CD276, CD40LG, CD8A, CADM3, CLDN19. & 0.0018 \\
\hline & ErbB signaling pathway & CDKN1A, AKT2, ELK1, SRC, CAMK2A... & 0.0037 \\
\hline & Ras signaling pathway & ABL1, AKT2, BCL2L1, ELK1, GNB2, RASA4B... & 0.0087 \\
\hline & cGMP-PKG signaling pathway & AKT2, ATP1A2, ATP2B2, GNA11, GNAI2... & 0.018 \\
\hline \multirow[t]{8}{*}{ Let-7b-3p } & Chemokine signaling pathway & AKT2, CX3CR1, CRKL, CRK, GNAI3, GNB4... & 0.044 \\
\hline & actin cytoskeleton & CDC42RKL, GNA13, ROCK1, ROCK2, SOS2 ... & 0.0086 \\
\hline & MAPK signaling pathway & CDC42, AKT2, CRKL, CRK, MECOM, RAP1B... & 0.00085 \\
\hline & AMPK signaling pathway & HMGCR, PFKFB3, AKT2, ELAVL1, RAB10... & 0.018 \\
\hline & Notch signaling pathway & DLL1, DLL4, HES1, JAG1, JAG2, MAML2, RBPJ... & 0.031 \\
\hline & Wnt signaling pathway & LRP6, ROCK2, WNT5B, CTNNB1, CUL1 ... & 0.0085 \\
\hline & TGF-beta signaling pathway & ROCK1, SP1, ACVR1, ACVR2B, BMPR2, CUL1... & 0.0085 \\
\hline & cGMP-PKG signaling pathway & AKT2, ATP2B1, GNA13, GNAI3, ROCK1 ... & 0.0036 \\
\hline \multirow[t]{2}{*}{ miR-454-5p } & Central carbon metabolism & AKT3, MAPK1, PDGFRA & 0.012 \\
\hline & Rap1 signaling pathway & AKT3, MAPK1, PDGFRA, TLN2 & 0.016 \\
\hline miR-1287-3p & Ras signaling pathway & ABL2, BCL2L1, RAB5A, EFNA3, FGF12 & 0.021 \\
\hline \multirow[t]{2}{*}{ miR-3121-5p } & Cell adhesion molecules & CLDN2, HLA-C, NRXN1, PTPRF, SELE, SDC1 & 0.017 \\
\hline & Cytokine receptor interaction & CXCL14, KIT, RELT, TNFRSF21, ACVR1... & 0.034 \\
\hline \multirow[t]{4}{*}{ miR-1256 } & ErbB signaling pathway & KRAS, EGF, PIK3CG, TGFA & 0.014 \\
\hline & Ras signaling pathway & KRAS, RAP1B, EGF, PIK3, CGRAC1, STK4 & 0.0093 \\
\hline & MAPK signaling pathwa & KRAS, MKNK2, RAP1B, TAB2, STK4 ... & $8.50 \mathrm{E}-5$ \\
\hline & Rap1 signaling pathway & KRAS, RAP1B, RAPGEF2, EGF, PIK3CG, RAC1 & 0.0068 \\
\hline miR-4685-3p & Inflammatory mediator regulation of TRP channels & ASIC1, ADCY3, ITPR2, MAP2K6 & 0.017 \\
\hline miR-5580-3p & ECM-receptor interaction & COL5A2, COL11A1, ITGA2, ITGA4, ITGA9 & 0.049 \\
\hline
\end{tabular}

* "P-value" refers to significance of function enrichment

Other host antiparasite strategies are the immune responses [40-42]. As well as apoptosis, we found that hsa-miR-3591-3p and hsa-miR-34b-5p regulate the epithelial immune responses. Several reports have shown that hsa-miR-34a regulate host immune responses in response to microbial infection. For example, hsa-miR-34a negatively regulate the innate immune response to viral infections by targeting interferon $\beta$ [43]. hsa-miR-3591-3p could increases interleukin 6 mRNA levels in THP-1 cells in response to lipopolysaccharide stimulation [44]. These data suggest that hsa-miR-3591-3p, hsa-miR-34b-5p, hsa-miR-942-5p, hsa-miR-18b-3p and hsa-miR-3976 may be involved in cell apoptosis or the immune responses to C. parvum infection. Hsa-miR-34b-5p, hsa-miR-3591-3p, 


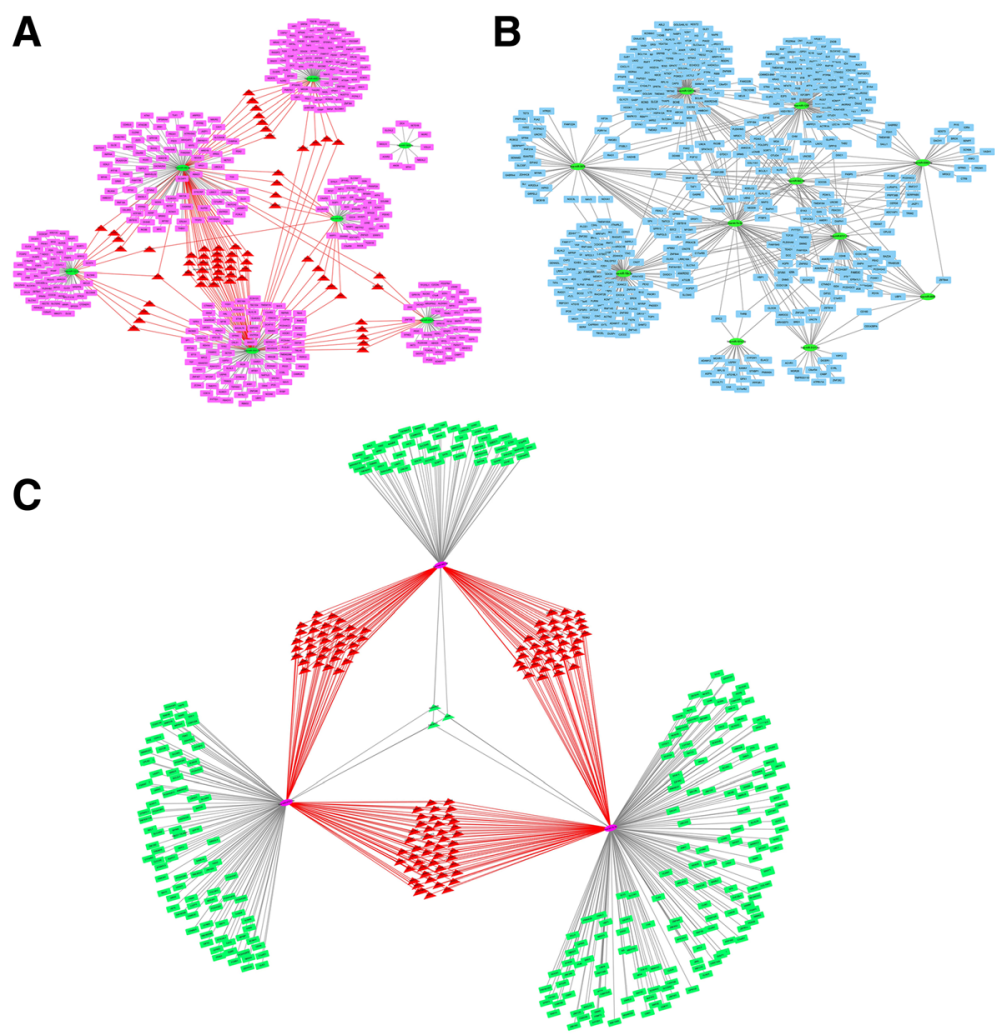

Fig. 4 miRNA-target gene network. Network of miRNAs and their target genes at $4 \mathrm{~h}$ after C. parvum infection (a). Network of miRNAs and target genes at $12 \mathrm{~h}$ after $\mathrm{C}$. parvum infection (b). Network of the key miRNAs and target genes at $12 \mathrm{~h}$ after $\mathrm{C}$. parvum infection (c). In the diagram of the miRNA-gene network, the ellipses represent the miRNAs, the rectangles represent the target genes, and triangles represent the key target genes, with the interaction between them are represented by lines. The crucial miRNAs has the highest connectivity which refers to the number of edges possessed by one miRNA. The key genes are the common target genes of differentially expressed miRNAs

hsa-miR-942-5p, hsa-let-7b-3p, and hsa-miR-6721-5p were also identified as the key miRNAs among the miRNAs differentially expressed after C. parvum infection. Therefore, the functions of hsa-let-7b-3p, hsa-miR-35913p, hsa-miR-34b-5p, hsa-miR-942-5p, hsa-miR-6721-5p, hsa-miR-18b-3p, hsa-miR-3976, and hsa-miR-3121-5p during C. parvum infection require further investigation.

There were some limitations to this study that should be addressed. First, the time points in the early phase of C. parvum infection was relatively limited and microRNA expression profile of HCT-8 cells in the later phase of C. parvum infection should be further analyzed to better understand the interplay between $C$. parvum and its host at the miRNA level. Second, we only assessed microRNA regulation in a single cell line, so the results should be confirmed with primary intestinal epithelial cells or another human intestinal epithelial cells line. Last, we should decrease the dose of oocysts used to infect the monolayer, because the high dose of oocysts may potentially have triggered a stress response in the host cell, the differentially regulated miRNA could reflect such a response and be unrelated to the actual $C$. parvum infection.

\section{Conclusions}

In this study, we determined the miRNA expression profile of human intestinal epithelial cells infected by $C$. parvum for the first time, and demonstrated that the dysregulation of these miRNAs may regulate the host's biological processes in response to C. parvum infection, including cell apoptosis and the immune responses. These findings extend our understanding of the miRNA regulatory network that operates during the host- $C$. parvum interaction. They also provide clues for further research into the regulatory mechanisms of miRNAs in human intestinal epithelial cells in response to C. parvum infection, which may identify potential targets for future C. parvum control strategies.

\section{Additional file}

Additional file 1: Figure S1. GO analysis of targets of differentially expressed miRNAs involved in the regulation of apoptotic processes and the immune response. (PDF $521 \mathrm{~kb}$ )

\section{Abbreviations}

DAVID: Database for annotation, visualization and integrated discovery; DMEM: Dulbecco's modified Eagle's medium; GO: Gene Ontology; 
KEGG: Kyoto encyclopedia of genes and genomes; SOCS: Suppressor of cytokine signaling

\section{Acknowledgments}

The authors thank Fuchang Yu, Jianke Cao, and Junqiang Li for their help with the animal infection experiment.

\section{Funding}

This study was supported by the Key Program of the National Natural Science Foundation of China (31330079), the National Natural Science Foundation of China $(31672548,31302079)$, and National Key Research and Development Program of China (2017YFD0501305, 2017YFD0500405). The funding bodies had no role in the design of the study, collection, analysis, interpretation of data and in the writing of the manuscript.

\section{Availability of data and materials}

All the data supporting our findings is contained within the manuscript. Full details of the miRNA microarray analyses were deposited in the Gene Expression Omnibus (GEO; http://www.ncbi.nlm.nih.gov/geo/) public database with the associated platform accession number GPL23111. The raw data are available through GEO under Accession: GSE95365. The entire microarray data set was MIAME compliant.

\section{Authors' contributions}

LoZ oversaw the entire project, including experimental design, reviewing the manuscript and submission, CW performed all experimental procedures including animal work, acquisition and analyses of data, drafting the manuscript, $\mathrm{LL}$ and $\mathrm{HZ}$ edited the manuscript and performed bioinformatic analyses, LuZ, ZZ, JH performed animal work and revised the manuscript, RW, FJ, SZ and CN performed bioinformatic and statistical analyses and contributed intellectually and critically to the manuscript. All the authors have read and approved the final manuscript.

\section{Ethics approval and consent to participate}

This research was conducted according to the Chinese Laboratory Animal Administration Act (1988) after the review and approval of its protocol by the Research Ethics Committee of Henan Agricultural University. No specific permits were required, and no endangered or protected species were involved.

\section{Consent for publication}

Not applicable.

\section{Competing interests}

The authors declare that they have no competing interests.

\section{Publisher's Note}

Springer Nature remains neutral with regard to jurisdictional claims in published maps and institutional affiliations.

\section{Author details \\ ${ }^{1}$ College of Animal Science and Veterinary Medicine, Henan Agricultural University, Zhengzhou 450002, People's Republic of China. ${ }^{2}$ International Joint Research Laboratory for Zoonotic Diseases of Henan, Zhengzhou 450002, People's Republic of China. ${ }^{3}$ College of Animal Science and Veterinary Medicine, Henan Institute of Science and Technology, Xinxiang 453003, People's Republic of China.}

Received: 7 February 2018 Accepted: 26 December 2018 Published online: 14 January 2019

\section{References}

1. Ryan U, Fayer R, Xiao L. Cryptosporidium species in humans and animals: current understanding and research needs. Parasitology. 2014;141(13):1667-85.

2. Hunter PR, Hadfield SJ, Wilkinson D, Lake IR, Harrison FC, Chalmers RM. Subtypes of Cryptosporidium parvum in humans and disease risk. Emerg Infect Dis. 2007;13(1):82-8.

3. Ryan U, Power M. Cryptosporidium species in Australian wildlife and domestic animals. Parasitology. 2012;139(13):1673-88.

4. Kotloff KL, Nataro JP, Blackwelder WC, Nasrin D, Farag TH, Panchalingam S, Wu Y, Sow SO, Sur D, Breiman RF, et al. Burden and aetiology of diarrhoeal disease in infants and young children in developing countries (the global enteric multicenter study, GEMS): a prospective, case-control study. Lancet. 2013;382(9888):209-22.

5. Striepen B. Parasitic infections: time to tackle cryptosporidiosis. Nature. 2013; 503(7475):189-91.

6. Bueno MJ, Pérez de Castro I, Malumbres M. Control of cell proliferation pathways by microRNAs. Cell Cycle. 2008;7(20):3143-8.

7. Kedde M, Agami R. Interplay between microRNAs and RNA-binding proteins determines developmental processes. Cell Cycle. 2008;7(7):899-903.

8. O'Connell RM, Rao DS, Chaudhuri AA, Baltimore D. Physiological and pathological roles for microRNAs in the immune system. Nat Rev Immunol. 2010;10(2):111-22.

9. Piedade D, Azevedo-Pereira JM. The role of microRNAs in the pathogenesis of herpesvirus infection. Viruses. 2016;8(6):156-87.

10. Zhou R, Hu G, Liu J, Gong AY, Drescher KM, Chen XM. NF-kappaB p65dependent transactivation of miRNA genes following Cryptosporidium parvum infection stimulates epithelial cell immune responses. PLoS Pathog. 2009;5(12):e1000681

11. Staedel C, Darfeuille F. MicroRNAs and bacterial infection. Cell Microbiol. 2013;15(9):1496-507.

12. Chen XM, Splinter PL, O'Hara SP, LaRusso NF. A cellular micro-RNA, let-7i, regulates toll-like receptor 4 expression and contributes to cholangiocyte immune responses against Cryptosporidium parvum infection. J Biol Chem. 2007:282(39):28929-38.

13. Hu G, Gong AY, Roth AL, Huang BQ, Ward HD, Zhu G, Larusso NF, Hanson $\mathrm{ND}$, Chen XM. Release of luminal exosomes contributes to TLR4-mediated epithelial antimicrobial defense. PLoS Pathog. 2013;9(4):e1003261.

14. Zhou R, Gong AY, Eischeid AN, Chen XM. miR-27b targets KSRP to coordinate TLR4-mediated epithelial defense against Cryptosporidium parvum infection. PLoS Pathog. 2012;8(5):e1002702.

15. Sato Y, Koshizuka T, Ishibashi K, Hashimoto K, Ishioka K, Ikuta K, Yokota SI, Fuiji N, Suzutani T. Involvement of herpes simplex virus type 1 UL13 protein kinase in induction of SOCS genes, the negative regulators of cytokine signaling. Microbiol Immunol. 2017:61(5):159-67.

16. Hu G, Zhou R, Liu J, Gong AY, Chen XM. MicroRNA-98 and let-7 regulate expression of suppressor of cytokine signaling 4 in biliary epithelial cells in response to Cryptosporidium parvum infection. J Infect Dis. 2010;202(1):125-35.

17. Sheedy FJ, Palsson-McDermott E, Hennessy EJ, Martin C, O'Leary JJ, Ruan O, Johnson DS, Chen Y, O'Neill LA. Negative regulation of TLR4 via targeting of the proinflammatory tumor suppressor PDCD4 by the microRNA miR-21. Nat Immunol. 2010;11(2):141-7.

18. Verdon R, Keusch GT, Tzipori S, Grubman SA, Jefferson DM, Ward HD. An in vitro model of infection of human biliary epithelial cells by Cryptosporidium parvum. J Infect Dis. 1997;175(5):1268-72.

19. Hu M, Xia M, Chen X, Lin Z, Xu Y, Ma Y, Su L. MicroRNA-141 regulates Smad interacting protein 1 (SIP1) and inhibits migration and invasion of colorectal Cancer cells. Dig Dis Sci. 2010:55(8):2365-72.

20. Saito Y, Liang G, Egger G, Friedman JM, Chuang JC, Coetzee GA, Jones PA. Specific activation of microRNA-127 with downregulation of the protooncogene BCL6 by chromatin-modifying drugs in human cancer cells. Cancer Cell. 2006:9(6):435-43.

21. Li R, Qian N, Tao K, You N, Wang X, Dou K. MicroRNAs involved in neoplastic transformation of liver cancer stem cells. J Exp Clin Cancer Res. 2010;29:169

22. Livak KJ, Schmittgen TD. Analysis of relative gene expression data using real-time quantitative PCR and the 2(-Delta Delta C(T)) method. Methods. 2001;25(4):402-8.

23. Agarwal V, Bell GW, Nam J, Bartel DP. Predicting effective microRNA target sites in mammalian mRNAs. elife. 2015;4:e05005.

24. Wong $N$, Wang $X$. miRDB: an online resource for microRNA target prediction and functional annotations. Nucleic Acids Res. 2015:43:D146-52.

25. Huang d W, Sherman BT, Lempicki RA. Bioinformatics enrichment tools: paths toward the comprehensive functional analysis of large gene lists. Nucleic Acids Res. 2009:37:1-13.

26. Shannon P, Markiel A, Ozier O, Baliga NS, Wang JT, Ramage D, Amin N, Schwikowski B, Ideker T. Cytoscape: a software environment for integrated models of biomolecular interaction networks. Genome Res. 2003;13(11): 2498-504.

27. Pahl MC, Derr K, Gäbel G, Hinterseher I, Elmore JR, Schworer CM, Peeler TC, Franklin DP, Gray JL, Carey DJ, et al. MicroRNA expression signature in humanabdominal aortic aneurysms. BMC Med Genet. 2012;5:25. 
28. Mokutani Y, Uemura M, Munakata K, Okuzaki D, Haraguchi N, Takahashi H, Nishimura J, Hata T, Murata K, Takemasa I, et al. Down-regulation of microRNA-132 is associated with poor prognosis of colorectal Cancer. Ann Surg Oncol. 2016;23(5):599-608.

29. Ji J, Shi J, Budhu A, Yu Z, Forgues M, Roessler S, Ambs S, Chen Y, Meltzer PS, Croce $C M$, et al. MicroRNA expression, survival, and response to interferon in liver cancer. N Engl J Med. 2009;361(15):1437-47.

30. Li J, Tan S, Kooger R, Zhang C, Zhang Y. MicroRNAs as novel biological targets for detection and regulation. Chem Soc Rev. 2014;43(2):506-17.

31. Chen XM, Levine SA, Tietz P, Krueger E, McNiven MA, Jefferson DM, Mahle M, LaRusso NF. Cryptosporidium parvum is cytopathic for cultured human biliary epithelia via an apoptotic mechanism. Hepatol. 1998;28(4):906-13.

32. McCole DF, Eckmann L, Laurent F, Kagnoff MF. Intestinal epithelial cell apoptosis following Cryptosporidium parvum infection. Infect Immun. 2000; 68(3):1710-3.

33. Chen XM, Gores GJ, Paya CV, LaRusso NF. Cryptosporidium parvum induces apoptosis in biliary epithelia by a Fas/Fas ligand-dependent mechanism. Am J Phys. 1999;277(1):599-608.

34. Widmer G, Corey EA, Stein B, Griffiths JK, Tzipori S. Host cell apoptosis impairs Cryptosporidium parvum development in vitro. J Parasitol. 2000;86(5):922-8.

35. Liu J, Deng M, Lancto CA, Abrahamsen MS, Rutherford MS, Enomoto S. Biphasic modulation of apoptotic pathways in Cryptosporidium parvum infected human intestinal epithelial cells. Infect Immun. 2009;77(2):837-49.

36. Cai Y, Chen H, Mo X, Tang Y, X X, Zhang A, Lun Z, Lu F, Wang Y, Shen J. Toxoplasma gondii inhibits apoptosis via a novel STAT3- miR-17-92-Bim pathway in macrophages. Cell Signal. 2014;26(6):1204-12.

37. Cai Y, Chen H, Jin L, You Y, Shen J. STAT3-dependent transactivation of miRNA genes following Toxoplasma gondii infection in macrophage. Parasit Vectors. 2013;6:356.

38. Fan N, Wang J. MicroRNA 34a contributes to virus-mediated apoptosis through binding to its target gene Bax in influenza a virus infection. Biomed Pharmacother. 2016;83:1464-70.

39. Yang D, Meng X, Xue B, Liu N, Wang X, Zhu H. MiR-942 mediates hepatitis C virus-induced apoptosis via regulation of ISG12a. PLoS One. 2014;9(4):e94501.

40. McDonald V, Korbel DS, Barakat FM, Choudhry N, Petry F. Innate immune responses against Cryptosporidium parvum infection. Parasite Immunol. 2013;35(2):55-64.

41. Pantenburg B, Dann SM, Wang HC, Robinson P, Castellanos-Gonzalez A, Lewis $D E$, White $A C$ Jr. Intestinal immune response to human Cryptosporidium sp. infection. Infect Immun. 2008;76(1):23-9.

42. Petry F, Jakobi V, Tessema TS. Host immune response to Cryptosporidium parvum infection. Exp Parasitol. 2010;126(3):304-9.

43. Witwer KW, Sisk JM, Gama L, Clements JE. MicroRNA regulation of IFN-beta protein expression: rapid and sensitive modulation of the innate immune response. J Immunol. 2010;184(5):2369-76.

44. Woo MY, Yun SJ, Cho O, Kim K, Lee ES, Park S. MicroRNAs differentially expressed in Behçet disease are involved in interleukin-6 production. J Inflamm. 2016;13:22.

\section{Ready to submit your research? Choose BMC and benefit from:}

- fast, convenient online submission

- thorough peer review by experienced researchers in your field

- rapid publication on acceptance

- support for research data, including large and complex data types

- gold Open Access which fosters wider collaboration and increased citations

- maximum visibility for your research: over $100 \mathrm{M}$ website views per year

At BMC, research is always in progress.

Learn more biomedcentral.com/submissions 